\title{
Sudden Onset Hemiplegia and Neglect: A Case Report of Type A Aortic Dissection Presenting as a Code Stroke
}

\author{
Thomas Gregg Pederson ${ }^{\mathrm{a}, \mathrm{c}}$, Yimage Ahmed ${ }^{\mathrm{b}}$, Joseph K. Maddry ${ }^{\mathrm{a}}$, Nurani M. Kester ${ }^{\mathrm{a}}$
}

\begin{abstract}
Stanford type A acute aortic dissection with ischemic stroke is a rare yet highly morbid presentation of sudden onset neurological symptoms. We present a case of a 57-year-old African American male brought to the emergency department with a witnessed syncopal episode, abdominal pain, right sided gaze preference, and left side weakness. Upon initiation of the hospital's "Code Stroke" response protocol, a computed tomography angiogram of the neck incidentally identified Stanford type A aortic dissection with extension into and occlusion of the brachiocephalic artery, occlusion of the right common carotid artery and right internal carotid artery, and dissection flap propagation into the origin of the left common carotid artery. Postoperative magnetic resonance imaging of the brain and the entire spine demonstrated multifocal cerebral infarcts, as well as T10 - L1 spinal infarct. Despite provision of multidisciplinary intensive care unit level of care, the patient failed to demonstrate clinically significant neurological recovery and was transitioned to comfort care after 3 weeks of hospitalization. This case highlights the importance of considering acute aortic dissection as a potential etiology of acute ischemic stroke through the use of computed tomography angiography, as this diagnosis carries profoundly different implications for the consideration of thrombolytic agents and other emergent treatment modalities.
\end{abstract}

Keywords: Aortic dissection; Acute ischemic stroke; CT angiography

\section{Introduction}

Acute aortic dissection (AAD) refers to the pathologic disruption of the aortic wall that occurs when blood enters and dis-

Manuscript submitted September 18, 2020, accepted November 19, 2020

Published online December 9, 2020

aDepartment of Emergency Medicine, University of Texas Health Science Center at San Antonio, San Antonio, TX 78229, USA

bDepartment of Surgery, University of Texas Health Science Center at San Antonio, San Antonio, TX 78229, USA

${ }^{c}$ Corresponding Author: Thomas Gregg Pederson, University of Texas Health Science Center at San Antonio, San Antonio, TX 78229, USA.

Email: Pedersont@uthscsa.edu

doi: https://doi.org/10.14740/jnr638 sects the intimal layer from the adventitial layer of the vessel [1]. AAD classically presents as sudden onset, sharp, ripping, or tearing quality chest pain that radiates to the back, between the scapula [2,3]. Risk factors in younger patients include connective tissue disorders such as Marfan syndrome as well as illicit drug use (i.e., cocaine), whereas hypertension, obesity, cigarette smoking, and ischemic heart disease are the predisposing factors in older patient populations $[1,2]$. The more commonly used AAD classification system is the Stanford system. Stanford type A dissections involve the ascending aorta proximal to the brachiocephalic artery, and these dissections necessitate surgical therapy. Stanford type B dissections involve only structures distal to the left subclavian artery branchpoint, and they are typically managed nonoperatively [3].

While chest pain is a predominant symptom of AAD, this "classic presentation" may only be present in half of patients. Other symptoms that should raise suspicion for AAD include hypertension, syncope, altered mental status, Horner syndrome, muffled heart sounds, and loss of peripheral pulses [3]. Patients with AAD are also at significant risk for acute ischemic stroke as a complication, which can occur as a result of global hypoperfusion from hypotension and from direct circulatory compromise via extension of the dissection flap into the cerebral vasculature [4].

The prevalence of acute ischemic stroke in patients with type A AAD is estimated at $6 \%$, and these patients account for $1.7 \%$ of all acute stroke cases $[4,5]$. AAD patients presenting with stroke are less likely to present with chest, abdominal, or back pain, and may instead present with syncope [5]. Given its variable clinical presentation, $15-43 \%$ of verified AAD remain missed during initial workup [6]. These factors contribute to the in-hospital mortality of $27.7 \%$ for AAD [7], and mortality increases $1-2 \%$ per hour from initial presentation to diagnosis and definitive management [8]. This case report emphasizes the importance of considering AAD as an etiology of acute ischemic stroke, as prompt identification of AAD can avoid erroneous administration of thrombolytic agents and expedite definitive surgical repair.

\section{Case Report}

The patient is a 57-year-old African American male with a past medical history of malignant hypertension with poor adherence to medical therapy and a long-standing history of heavy smoking. He was brought to the emergency department of a large academic teaching hospital by Emergency Medical Ser- 
Table 1. Presenting Initial NIH Stroke Scale (NIHSS) Score as Determined by Consulting Neurology Stroke Service.

\begin{tabular}{ll}
\hline Level of consciousness & 0 \\
Level of consciousness questions & 0 \\
Level of consciousness commands & 0 \\
Extraocular movements & 2 \\
Visual fields & 2 \\
Facial palsy & 0 \\
Left arm motor drift & 4 \\
Right arm motor drift & 0 \\
Left leg motor drift & 4 \\
Right leg motor drift & 0 \\
Limb ataxia & 2 \\
Sensation & 0 \\
Language/aphasia & 0 \\
Dysarthria & 1 \\
Extinction/inattention & 2 \\
Total & 17 \\
\hline
\end{tabular}

This patient's NIHSS is 17 , which is categorized as severe. NIH: National Institutes of Health.

vices (EMS) at 11:59 am, $1 \mathrm{~h}$ after experiencing a witnessed syncopal episode while at work.

EMS reported that the patient had abdominal pain and headache and was observed to be lapsing in and out of consciousness during transport. The hospital's "Code Stroke" response protocol was activated in the field by EMS for findings of left-sided facial droop and left upper extremity weakness; and the patient was triaged directly to computed tomography (CT) upon arrival to the hospital. During primary examination, the patient was in moderate distress but responsive with an initial National Institutes of Health (NIH) Stroke Scale (NIHSS) score of 17, as depicted in Table 1. His NIHSS was remarkable for left sided weakness, dysarthria, right sided gaze preference. He was afebrile with a heart rate of 53 beats per minute, non-invasive blood pressure (NIBP) of 92/64 mm $\mathrm{Hg}$ on the right arm, respiratory rate of 18 breaths per minute, oxygen saturation $97 \%$ on room air, and point-of-care glucose of $150 \mathrm{mg} / \mathrm{dL}$.

CT of the head 9-min after arrival was negative for hemorrhagic stroke. Fourteen minutes after arrival, CT angiogram of the head and neck demonstrated occlusion of the right common carotid artery and cervical right internal carotid artery, a $4 \times 3 \mathrm{~mm}$ saccular aneurysm arising from the right supraclinoid internal carotid artery, and a partially visualized dissection of the ascending aortic arch with a dissection flap dissecting into the brachiocephalic artery. Arterial and central venous access were obtained, and repeat vitals were remarkable for blood pressure discrepancy of 210/66 mm Hg by invasive left radial arterial line and $115 / 82 \mathrm{~mm} \mathrm{Hg}$ by right arm NIBP. The patient was placed on esmolol, nicardipine, and nitroglycerin drips for blood pressure and heart rate control, and he was given fentanyl and lorazepam intravenous (IV)

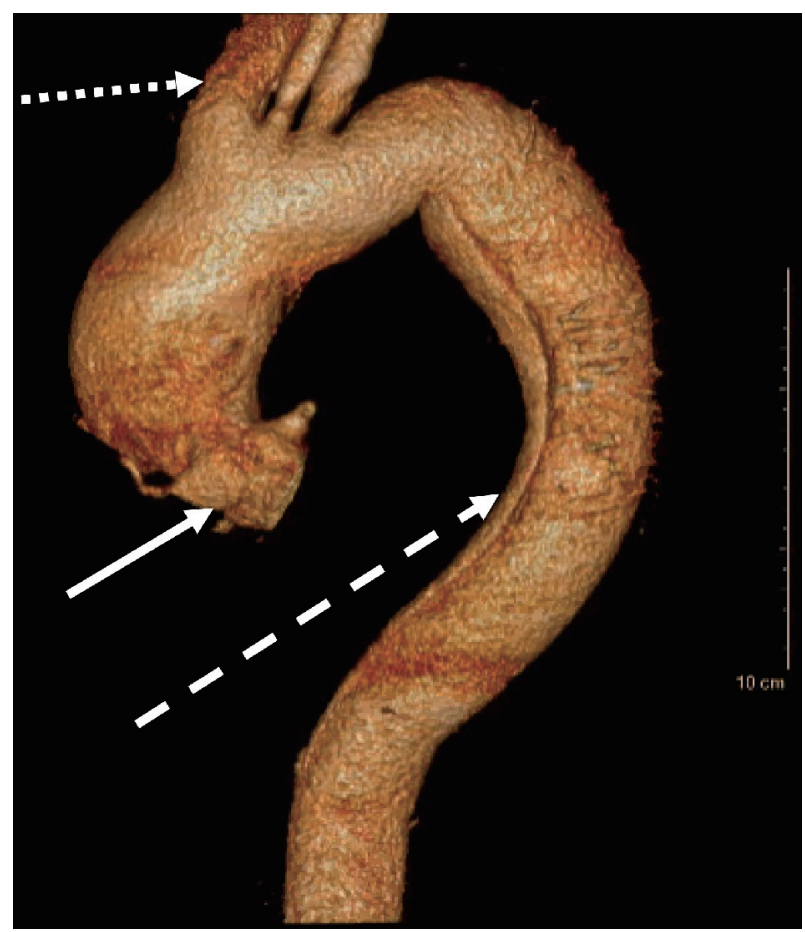

Figure 1. CT angiogram thoracic aorta three-dimensional (3D) reconstruction demonstrating Stanford type A AAD at the aortic root (solid white arrow), with extension into the innominate artery (dotted white arrow) and distal extension to the descending thoracic aorta (dashed white arrow). CT: computed tomography.

pushes for agitation.

Follow-up CT angiogram of the thoracic and abdominal aorta $1 \mathrm{~h}$ after arrival revealed a Stanford type A aortic dissection originating at the aortic root, with distal extension to the left external iliac artery and involvement of the mesenteric and renal vasculature (Fig. 1). He was emergently taken to the operating room (OR) for operative repair of the Stanford type A dissection which consisted of cannulation for cardiopulmonary bypass, resection and graft replacement of the ascending aorta and hemiarch, and primary repair of aortic arch penetrating atherosclerotic ulcer.

There were no remarkable intraoperative complications, and the patient was taken postoperatively to the intensive care unit (ICU) while intubated and sedated. The patient was taken off sedation medications on postoperative day 1 , and he was noted to have a Richmond Agitation and Sedation Scale (RASS) of -5. His postoperative neurological exam was remarkable for spontaneous right upper extremity movement, briskly reactive right pupil to light, and a sluggishly reactive left pupil to light. Repeat CT head performed at $37 \mathrm{~h}$ after initial presentation demonstrated bilateral posterior cerebral, bilateral middle cerebral artery (MCA), left MCA, and left anterior cerebral artery watershed ischemic strokes, without cerebral edema, hemorrhage, or herniation. Simultaneous electroencephalogram (EEG) was concerning for diffuse severe cerebral dysfunction. The patient attained at best Glasgow Coma Scale of Eye (E) 2-Verbal (V) 1T-Motor (M) 5 by hospital day 3. Exam on day 3 was remarkable for interval 


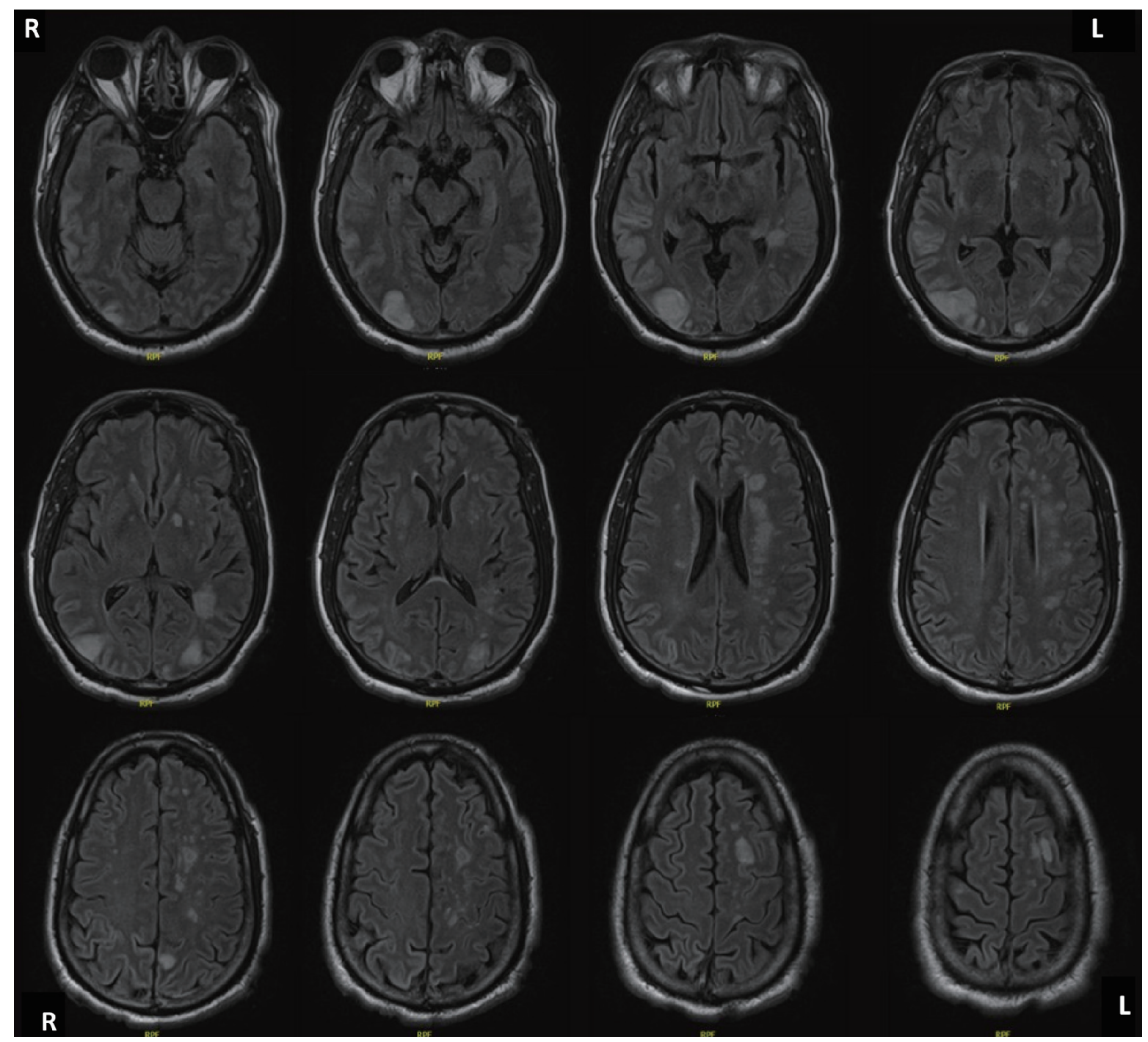

Figure 2. MRI brain without contrast, axial T2 weighted imaging, demonstrating diffuse regions of increased relaxation time in the left periventricular white matter, left frontal, right temporal, left cerebellar hemisphere, left parietal, and bilateral occipital lobes, and left hypothalamus, consistent with global watershed ischemia and infarction territories. MRI: magnetic resonance imaging.

worsening of his motor exam, with localization to pain in the left upper extremity, withdrawal to pain in the right upper extremity, and no movement in the bilateral lower extremities. A follow-up magnetic resonance imaging (MRI) brain with T2wSTIR sequence obtained on hospital day 4 demonstrated recent multifocal infarcts, as well as several scattered punctate foci of age-indeterminate micro-hemorrhages (Fig. 2). Simultaneous MRI of the entire spine demonstrated cord infarction at the level of T10 - L1. CT of the head on hospital day 9 re-demonstrated initial findings of multifocal infarcts with left frontal, parietal, and occipital predominance; no bleed, no herniation, and no hemorrhagic transformation.

The patient was followed by neurology critical care and neurology stroke services for an additional 2 weeks. Unfortunately, he did not regain any additional neurological function and was unable to wean from mechanical ventilation. The patient was transitioned to hospice care on hospital day 22 , he was terminally extubated on hospital day 23 per advanced care directive, and he died 24 days after initial presentation.

\section{Discussion}

Aortic dissection classically presents with ripping chest pain or abdominal pain, and the diagnosis of ischemic stroke is appreciated secondarily [3]. However, a retrospective chart review found that $29 \%$ of patients with AAD presented with predominantly neurologic symptoms, and these patients were significantly less likely to report symptoms of pain [2]. A wide variety of neurological symptoms have been documented in patients with aortic aneurysms. In addition to our patient case report, other reported neurologic symptoms presented in patients with AAD include dysarthria with mild left limb paresis with Babinski sign, aphasia with severe right limbs paresis, 
and a third case with aphasia and right limb paresis [9]. Neurologic symptoms were almost twice as prevalent in patients with dissection into the supra-aortic vessels. This complication occurred in almost half of Stanford type A AADs and involved the carotid vasculature $81.2 \%$ of the time, as was seen in this patient [2]. While most neurological complications are attributed to the AAD propagation into the supra-aortic vessels, a combination of focal and global ischemia may also result from sustained hypotension secondary to cardiac tamponade, exsanguination into the pleural or retroperitoneal spaces, or frank aortic rupture [10]. The variability of ischemic insults attributable to AAD emphasizes the importance of considering the diagnosis for all patients who present with stroke symptoms.

Recent guidelines for acute stroke management support the use of thrombolytic agents for patients presenting within $4.5 \mathrm{~h}$ of ischemic stroke symptoms without evidence of hemorrhage or signs of extensive infarction on non-contrast CT $[11,12]$. In these authors' opinion, these guidelines risk delaying the recognition of AAD until after the administration of thrombolytics. This risk is not theoretical, as demonstrated by a recent single-center retrospective chart review of 2,874 "Code Stroke" activations in which one in 200 patients had AAD [13]. In this case report, the patient's profound initial neurologic deficits prompted a "Code Stroke" activation, in which a non-contrast head CT as well as CT angiography head and neck to the level of the aortic arch were performed, and a near-miss administration of thrombolytics to the patient with type A AAD was avoided. The case highlights the utility of CT angiography in patients presenting with any stroke symptoms. Furthermore, these authors advocate for performance of CT angiography to the level of the aortic arch in these situations [13].

In situations where patients with neurological deficits do not trigger a "Code Stroke" activation and are not directly triaged to CT imaging suites, a portable anteroposterior (AP) chest X-ray should be obtained to quickly identify features such as a mediastinal widening and apical pleural capping that would prompt further investigation of possible AAD [14]. Clinicians should also perform a focused physical exam of all extremities, paying special attention to peripheral pulse quality, skin temperature, and blood pressure discrepancies that could indicate compromised arterial blood flow from propagating aortic dissection flaps [14].

This patient did not survive, and this unfortunate fact is consistent with another retrospective chart review in which AAD patients presenting with stroke symptoms had twice the mortality as those without stroke symptoms [15]. Ultimately, the diagnosis of AAD with concomitant ischemic stroke has profound implications for which emergent treatment modalities are available. A prompt identification of patients with both of these problems can reduce the risk of unintended patient harm and allow for a more accurate prognostication of patient mortality.

\section{Acknowledgments}

None to declare.

\section{Financial Disclosure}

None to declare.

\section{Conflict of Interest}

None to declare.

\section{Informed Consent}

Not applicable.

\section{Author Contributions}

JKM and NMK supervised and finalized the manuscript; YA and TGP participated in the literature review, writing, and editing of this manuscript.

\section{Data Availability}

The authors declare that data supporting the findings of this study are available within the article.

\section{References}

1. Johnson G, Prince L. Aortic dissection and related aortic syndromes. In: Tintinalli's emergency medicine: a comprehensive study guide 9th ed. McGraw-Hill Education; 2020: p. 412-415.

2. Gaul C, Dietrich W, Friedrich I, Sirch J, Erbguth FJ. Neurological symptoms in type A aortic dissections. Stroke. 2007;38(2):292-297.

3. Levy D, Goyal A, Grigorova Y, Le JK. Aortic dissection. In: StatPearls. StatPearls Publishing; 2020. Accessed August 21, 2020. http:/www.ncbi.nlm.nih.gov/books/ NBK441963/.

4. Koga M, Iguchi Y, Ohara T, Tahara Y, Fukuda T, Noguchi $\mathrm{T}$, Matsuda H, et al. Acute ischemic stroke as a complication of Stanford type A acute aortic dissection: a review and proposed clinical recommendations for urgent diagnosis. Gen Thorac Cardiovasc Surg. 2018;66(8):439-445.

5. Bossone E, Corteville DC, Harris KM, Suzuki T, Fattori R, Hutchison S, Ehrlich MP, et al. Stroke and outcomes in patients with acute type A aortic dissection. Circulation. 2013;128(11 Suppl 1):S175-179.

6. Hagan PG, Nienaber CA, Isselbacher EM, Bruckman D, Karavite DJ, Russman PL, Evangelista A, et al. The International Registry of Acute Aortic Dissection (IRAD): new insights into an old disease. JAMA. 2000;283(7):897903.

7. Pacini D, Di Marco L, Fortuna D, Belotti LM, Gabbieri D, Zussa C, Pigini F, et al. Acute aortic dissection: epidemiology and outcomes. Int J Cardiol. 2013;167(6):2806- 
2812.

8. Ohle R, Kareemi HK, Wells G, Perry JJ. Clinical Examination for Acute Aortic Dissection: A Systematic Review and Meta-analysis. Acad Emerg Med. 2018;25(4):397412.

9. Pastuszak Z, Stepien A, Kordowska J, Rolewska A, Galbarczyk D. Brain strokes related to aortic aneurysma - the analysis of three cases. Open Med (Wars). 2017;12:58-61.

10. Blanco M, Diez-Tejedor E, Larrea JL, Ramirez U. Neurologic complications of type I aortic dissection. Acta Neurol Scand. 1999;99(4):232-235.

11. Wannamaker R, Buck B, Butcher K. Multimodal CT in Acute Stroke. Curr Neurol Neurosci Rep. 2019;19(9):63.

12. Boulanger JM, Lindsay MP, Gubitz G, Smith EE, Stotts G, Foley N, Bhogal S, et al. Canadian stroke best practice recommendations for acute stroke management: prehospital, emergency department, and acute inpatient stroke care, 6th edition, Update 2018. Int J Stroke. 2018;13(9):949-984.

13. Guglielmi V, Groeneveld NS, Posthuma L, Groot AE, Majoie CB, Talacua H, Kaya A, et al. Aortic dissection masquerading as a code stroke: A single-centre cohort study. Eur Stroke J. 2020;5(1):56-62.

14. Bressler M, Klein E, Shayowitz M, Gupta K, Delman A. A case of late diagnosis of acute aortic dissection involving the carotid arteries in a patient presenting with signs of acute ischemic stroke. Am J Case Rep. 2020;21:e919271.

15. Dumfarth J, Kofler M, Stastny L, Plaikner M, Krapf C, Semsroth S, Grimm M. Stroke after emergent surgery for acute type A aortic dissection: predictors, outcome and neurological recovery. Eur J Cardiothorac Surg. 2018;53(5):1013-1020. 